\title{
Self diffusion of polymer-like micelles
}

\section{A. OTT, N. MORIE, W. URBACH, J.P. BOUCHAUD and D. LANGEVIN}

Laboratoire de Physique Statistique, Ecole Normale Supérieure, 24 rue Lhomond, 75231 Paris cedex 05, France

We present a short review of fluorescence recovery (FRAP) experiments done with polymer-like micelles, allowing the study of self-diffusion in these media. The observed behavior is varied, specially in the semi-dilute regime where one can observe reptation processes interrupted by micelle breakage and recombination, and in some cases accelerated diffusion.

Cet article est une courte revue portant sur l'étude de l'autodiffusion dans les solutions de micelles polymériques par recouvrement de fluorescence (FRAP). Le comportement observé est très varié, en particulier dans la région semi-diluée, où l'on observe un processus de reptation interrompu par les coupures et les recombinaisons des micelles, qui conduit dans certains cas à une diffusion accélérée. 


\section{Introduction}

Diffusion processes result from the brownian motion of molecules or larger particles in a medium. Self-diffusion is generally investigated with "tracer" molecules, i.e. test molecules added in very small amounts to the medium. In classical quasi-elastic light scattering (QELS), the nature of the investigated diffusion processes is different ; with solutions, the technique is sensitive to concentration fluctuations of the different molecular species; the corresponding time relaxation is characterized by a diffusion coefficient called "mutual" $\mathrm{D}_{m}$. The difference between mutual and self diffusion coefficients $D_{m}$ and $D_{s}$ can be very large, for instance close to a critical consolute point where the amplitude of the concentration fluctuations diverges and $D_{m}$ goes to zero, whereas $D_{s}$ remains finite. $D_{s}$ is sensitive to "connectivity" fluctuations and goes to zero at a percolation threshold where $D_{m}$ remains finite ${ }^{(1)(2)}$. Cases where $D_{s}$ and $D_{m}$ are very different include entangled polymer solutions. The polymer forms a mesh of characteristic size $\xi$, and the observed relaxation of concentration fluctuations frequently involves the breathing mode of the mesh $D_{m}=D_{0} / \xi\left(D_{0}=k T / 6 \pi \eta, k=\right.$ Boltzmann constant, $T$ $=$ absolute temperature, $\eta=$ viscosity of the medium). The self-diffusion process involves the motion of a single polymer chain in the mesh, the so called "reptation process", which is much slower : $D_{S} \approx D_{0}$ a/L $/ L^{2}$ where $a$ is the monomer size and $L$ the polymer chain length (3). Typically $\mathrm{a} \sim 10 \AA, \mathrm{L} \sim 1 \mu \mathrm{m}$ and $\xi \sim 100 \AA$, leading to $\mathrm{D}_{\mathrm{m}} / \mathrm{D}_{\mathrm{s}} \sim 10^{5}$.

In QELS experiments, it is sometimes possible to probe time scales short enough, so that the diffusing species do not see a structural change in their environment. The diffusing motion is not affected by the "memory" effects related to the time evolution of the environment, and the measured coefficient is the self-diffusion one (4). In other cases, and at longer time scales, for instance in dispersions of polydisperse spheres, part of the scattering is "incoherent"; this incoherent scattering is associated with the polydispersity fluctuations. When the polydispersity is small, the corresponding relaxation process is quasi-exponential and gives the mean value of the self-diffusion coefficient (5). These two methods for the measurement of $\mathrm{D}_{\mathrm{s}}$ are unfortunately limited to a very small number of systems.

The methods commonly used to probe self-diffusion are :

- Macroscopic studies of the diffusion of different types of tracers : radioactive, dye, ... These methods are time consuming : for $D_{\mathrm{S}} \sim 10^{-11} \mathrm{~m}^{2} / \mathrm{s}$ and a distance travelled $\mathrm{R} \sim 1 \mathrm{~mm}$, the diffusion time is $10^{4} \mathrm{sec}$.

- Microscopic studies with optical devices such as Forced Rayleigh Scattering (5), fluorescence recovery after photobleaching (FRAP) (6), in which a tracer molecule (a photochromic molecule in the first case, a bleachable fluorescent molecule in the second) diffuses over lenght scales of order of microns ; the diffusion times are thus reduced to $10^{-2} \mathrm{~s}$ for $\mathrm{D} \sim 10^{-11} \mathrm{~m}^{2} / \mathrm{s}$. There are only few known photochromic molecules, which are not always 
soluble in the medium to be studied. The choice of fluorescent molecules is much larger, and the FRAP method is much more widely used, in particular in biology where frequently some constituents of the medium are themselves fluorescent and able to be bleached.

- Methods in which no tracers are added, such as spin echo NMR, where the diffusion coefficients of all the different molecular species can in principle be studied.

In surfactant systems, these methods have been widely used as a probe of the structure. It is not easy to distinguish between for instance micellar cubic and bicontinuous cubic phases, on the basis of such purely structural studies as X-ray or neutron scattering experiments. However, the self-diffusion in the surfactant layer is very different in the two cases: slow in the micellar structure, faster in the bicontinuous structure where the probe can travel freely along the layer (7). Similarly, the structural data on concentrated microemulsions are very similar for droplets and bicontinuous structures (where the surfactant layer separates continuous oil and water phases as in the bicontinuous cubic phases). The self-diffusion method, being dynamic in nature, cannot however be pushed too far. For instance, it has been found that the surfactant self-diffusion coefficient measured with NMR in bicontinuous microemulsions phases is $\mathrm{D}_{\mathrm{s}} \sim 10^{-10} \mathrm{~m}^{2} / \mathrm{s}$, i.e. only six times smaller than in pure water ${ }^{(8)}$. This could lead to the conclusion that the medium is structureless and close to a molecular mixture of oil, water and surfactant. Forced Rayleigh experiments done with a surfactant spiropyran probe embedded in the surfactant layer of the same microemulsions showed that $D_{s} \sim 10^{-11} \mathrm{~m}^{2} / \mathrm{s}$. This value is of the right order of magnitude for a diffusion process in a two-dimensional surfactant layer (9). The discrepancy between the two values of $D_{s}$ can be traced to the exchange of surfactant (and probe) molecules between the surfactant layer and the oil and water phases. The measured $D_{S}$ is then the average over the different residence times of the diffusion coefficients in the different media. In most cases, the residence times are not precisely known, but they can be changed easily in the techniques using probes, by changing these probes. For instance, in order to measure the self-diffusion coefficient in the surfactant layer, FITC molecules (fluorescein isothiocyanate) with branched alkyl chains are frequently used in FRAP measurements. It is known that the residence time of the probe in the surfactant layer increases by a factor 3 if the number of carbon atoms in the alkyl chain increases by one (10). If the measured $D_{s}$ does not change with the different probes, one can safely assume that these probes remain embedded in the surfactant layer most of the time and that the measured coefficient corresponds to this limit. By using this procedure, we were able to show unambiguously that the $\mathrm{L}_{3}$ phase of aqueous solution of aminoxide surfactants was not a dispersion of disc-like micelles, but rather a "sponge" phase with interconnected surfactant layers, although previous experiments using neutron and light scattering, QELS, transient electric birefringence were unable to discriminate between the two possible structures ${ }^{(11)}$. 
In the present paper, we will present a short review of experiments done with polymerlike micelles, where the study of self-diffusion showed a considerable variety of behaviour. These systems have also very interesting rheological behavior : systems containing less than $1 \mathrm{wt} \%$ surfactant can be viscoelastic. Here, the self-diffusion does not only allow the study of the structure, but also of the characteristic relaxation mechanisms involved in the viscoelastic behavior.

\section{Polymer-like micelles}

The shape of surfactant aggregates in dilute aqueous solutions is mainly determined by the packing conditions of the surfactant molecules. This has been rationalized by Tanford (12) and later Israelachvili, Mitchell and Ninham (13) who introduced a packing parameter $v / \Sigma \mathbf{l}_{\mathbf{c}}$, where $v$ is the volume occupied by the surfactant hydrophobic part, $\Sigma$ the area per surfactant molecules and $\mathrm{l}_{\mathrm{c}}$ the extended chain length of the hydrophobic part. When $\mathrm{v} / \Sigma \mathrm{l}_{\mathrm{c}}<1 / 3$, spherical micelles are formed, when $\mathrm{v} / \Sigma \mathrm{l}_{\mathrm{c}} \sim 1$ lamellar phases. If $1 / 3<\mathrm{v} / \Sigma \mathrm{l}_{\mathrm{c}}<1 / 2$, cylindrical micelles are formed. An easy way to go from spherical to cylindrical micelles is to decrease $\Sigma$. This is conveniently achieved by adding salt to the aqueous solution: salt screens the electrostatic repulsions between the surfactant polar parts and allows them to come closer.

Although cylindrical micelles are frequently observed, they do not grow very long except in particular cases, and especially when cationic surfactants are used (14). The growth also depends on the nature of the salt molecules. Cetyltrimethylammonium bromide (CTAB) micelles grow faster with added sodium salicylate than with potassium bromide. Reverse micelles (surfactant in an organic solvent) also can grow very long in some cases, when trace water is added (15). The growth processes are not yet fully understood.

When the length of the micelle exceeds the persistence length $l_{p}$, it becomes flexible and worm-like as evidenced by electron microscopy (16). If $\mathrm{K}$ is the bending modulus of the surfactant layer, it has been shown that ${ }^{(17)}$ :

$$
\mathrm{l}_{\mathrm{p}}=\mathrm{aK} / \mathbf{k T}
$$

where $a$ is a molecular length. In known systems $l_{p} \sim 100-150 \AA$ (18)(19).

The average micelle length $\mathbf{L}$ increases with the surfactant volume fraction $\phi$. Many theoretical models were proposed to account for this variation : assuming a dynamic equilibrium between the micelles of different sizes, most models find $\mathbf{L} \sim \phi^{1 / 2}$. When $\mathbf{L}$ is large, the polydispersity (proportional to $\mathbf{L}$ ) is very large. In the Cates model, one assumes that scission can occur anywhere along the chain with equal probability $\mathrm{C}$ per unit length and that two chains 
may fuse in a way independent of their size ${ }^{(20)}$. The equilibrium size distribution is then found to be :

$$
\mathrm{P}(\mathrm{L}) \sim \mathrm{L} / \mathrm{L} \exp (-\mathrm{L} / \mathrm{L})
$$

with

$$
\mathbf{L} \sim \phi^{1 / 2} \exp (\mathrm{E} / 2 \mathrm{kT})
$$

where $\mathrm{L}$ is the micelle length and $\mathrm{E}$ is the scission energy. Typically $\mathrm{E} \sim 20 \mathrm{kT}$, i.e. about the number of monomers in the micellar end caps times $k T$ (14). The average scission or breaking time is :

$$
\tau_{\mathrm{b}}=1 / \mathbf{C L}
$$

For sufficiently large $\phi$, the micelles entangle like polymers and form a mesh of size $\xi \sim \phi^{v /(1-3 v)}$ ( the exponent $v$ is such as $\xi \sim\left(L_{b} / l_{p}\right)^{v}, L_{b}$ being the chain length in the blob of size $\xi$ ). For polymers in a good solvent $v=3 / 5$, leading to $\xi \sim \phi^{-0.75}$ in good agreement with both light scattering and elastic modulus data on polymer-micelles $(21)(22)$.

However, the dynamic behavior of entangled polymer-micelles at time scales longer than $\tau_{b}$ differs markedly from usual polymers. Because the micelles can break and recombine, their size change constantly and an effective monomer diffusion needs to be considered; as a result, the diffusion process is accelerated. According to Cates, when the scission time $\tau_{b}$ is smaller than the reptation time $\tau_{\text {rep }}$, a given monomer (or probe) in a micelle performs independent motions of characteristic arc length $\left(D_{c}(L) \tau\right)^{1 / 2}$ such that $C \tau\left(D_{c}(L) \tau\right)^{1 / 2}=1$ where $D_{c}$ is the curvilinear diffusion coefficient $D_{c}(L)=D_{0} / L$. This leads to $\tau=\tau_{b}^{2 / 3} \tau_{\text {rep }}^{1 / 3}(L)$ and to an effective diffusion coefficient :

$$
D_{\text {cates }} \sim\left(\tau_{\text {rep }} / \tau_{\mathrm{b}}\right)^{1 / 3} D_{\text {rep }}(L)
$$

Note that this argument assumes that the probe always sits on a chain of length close to L. The concentration dependence can be obtained by considering, as for usual polymers, that the basing unit in a semi-dilute polymer solution is the blob of size $\xi(3)$; remembering that $\mathbf{L}$ $\sim \phi^{1 / 2}$ and that $\tau_{\mathrm{b}} \sim 1 / \mathbf{L} \sim \phi^{-1 / 2}$, Cates obtained:

$$
\mathrm{D}_{\text {cates }} \sim \phi^{-1.58}
$$


A more rigourous self-consistent calculation, where excluded volume effects are considered in the derivation of the equilibrium size distribution, leads to $L \sim \phi^{0.6}, D_{\text {rep }}(L) \sim \phi^{3}$ and $D_{\text {cates }} \sim \phi^{1.70}$ (with $v=0.59$ ) $(20)$.

In the case where $\tau_{b}>\tau_{\text {rep }}, D_{s}$ would be equal to $D_{\text {rep }}$. For a mean chain :

$$
\mathrm{D}_{\text {rep }}(\mathbf{L}) \sim \phi^{-11 / 4}
$$

The $\phi$ dependence is stronger in this case. Moreover, each micelle will contribute with its own diffusion coefficient and because the polydispersity is large, the distribution of relaxation times will be very broad.

\section{Experimental results.}

The experimental set up (fig. 1) is described in ref. 23. In brief, a laser beam is split into two beams which produce interference fringes on the sample. The fringe spacing $i$ can be varied between 5 and $100 \mu \mathrm{m}$. The fluorescence of the probes is bleached in the light fringes. The fluorescence of the illuminated region, detected with a collecting lens and a photomultiplier, thus decreases. The system of fringes is then replaced by a similar system whose intensity has been decreased by a large factor in order to prevent further bleaching. Because of the diffusion

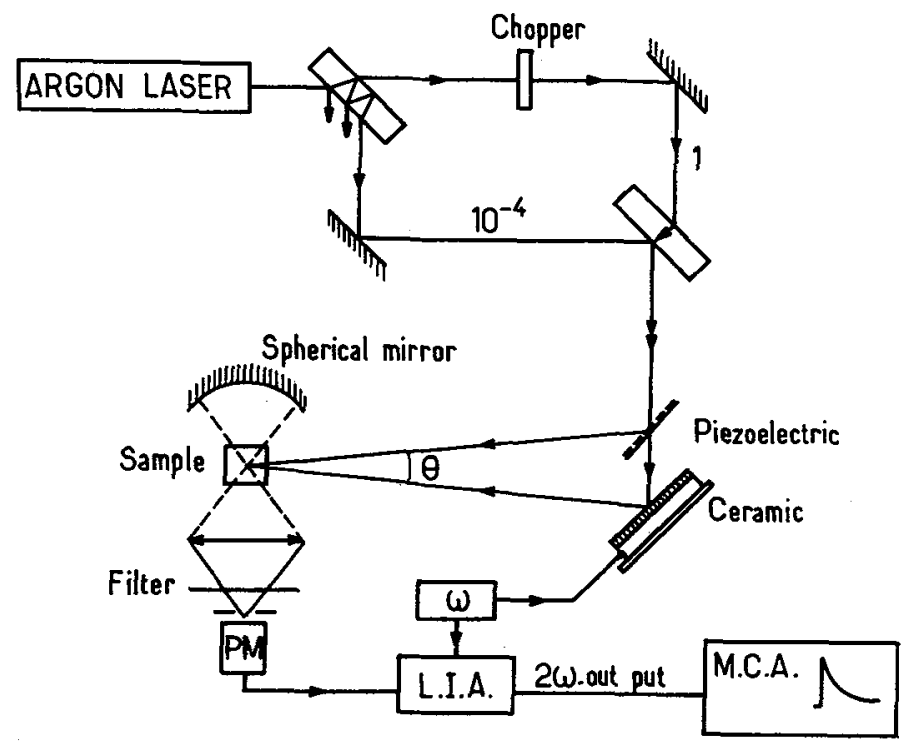

Figure 1. Experimental set-up. The upper glass plates produce perfectly superimposed light beams of intensities in the ratio $10^{4}$. LIA : lock-in amplifier, MCA : multichannel analyser. See text for details. 
of fluorescent probes from the dark to the light fringes, a portion of the fluorescent intensity is recovered. The recovery is exponential in the case of a single diffusing species, and the time of recovery is $\tau=1 / \mathrm{D}_{\mathrm{S}} \mathrm{q}^{2}$ where $\mathrm{q}=2 \pi / \mathrm{i}$. This transient signal is observed on the top a large constant fluorescent background. In order to suppress the background and to improve the signal-to-noise ratio, one of the mirrors is mounted on a piezo electric device to produce a modulation of the position of the fringes, and the fluorescence intensity modulations are directly analysed with a lock-in amplifier. A typical signal is shown in fig. 2 . It corresponds to CTAB micelles in the semi-dilute domain. Despite of the polydispersity, the recovery signal is exponential within instrumental accuracy. This means that $\tau_{\mathrm{b}}<\tau_{\text {rep }}$ and that the diffusion process is a succession of reptation steps followed by breakage and recombination of the micelles. For $D_{s} \leq 10^{-11} \mathrm{~m}^{2} / \mathrm{s}$ and $\mathrm{i} \geq 10 \mu \mathrm{m}$ this means that $\tau_{\mathrm{b}} \leq 1 \mathrm{sec}$. This is in agreement with $\tau_{b}$ measurements ${ }^{(24)}$.

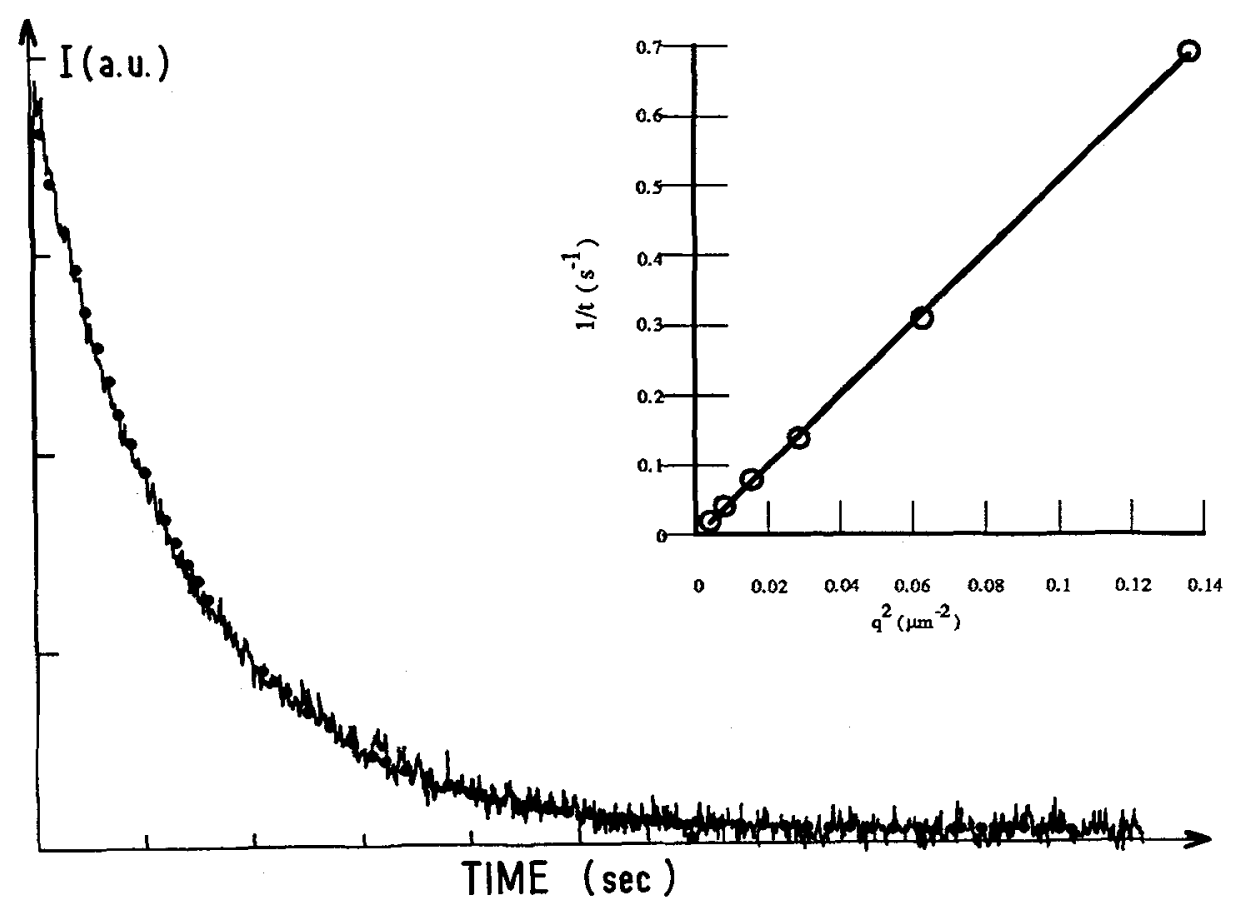

Figure 2. Typical signal. The dots show the fit with an exponential curve. Insert : inverse measured diffusion time versus $\mathrm{q}^{2}$. 
Data for CTAB at different salinities are shown on fig. 3. The low concentration region corresponds to small micelles which are not entangled. Above an entanglement concentration, smaller for larger salt concentrations, the diffusion coefficient follows a power law $D_{s} \sim c^{-\alpha}$ as predicted by eq. 6 . However, the exponent $\alpha$ is larger than the Cates exponent for the small salinities. This discrepancy is due to the fact that the surfactant is ionic; if the salt concentration $c_{\mathrm{S}}$ is small and comparable to the surfactant concentration $c$, an increase in c produces an effect similar to the addition of salt, namely a decrease of $\Sigma$ and an increase of the value of the surfactant parameter. The micellar length increases therefore faster than $\mathrm{c}^{1 / 2}$. This problem was considered theoretically by Safran et al. (25) and the results are in qualitative agreement with the data.

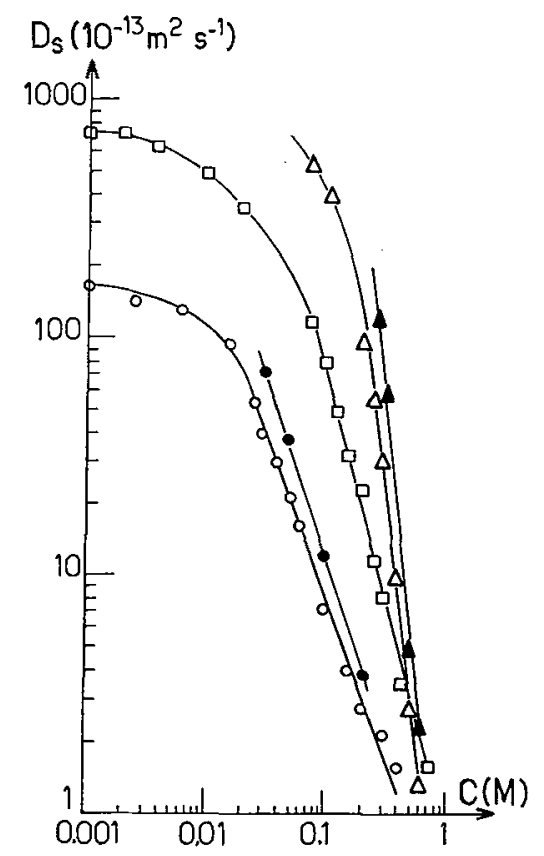

Figure 3. $\log -\log$ plot of $\mathrm{D}_{\mathrm{s}}$ versus c. Values of the slopes are : open circles $(0.25 \mathrm{M}$, $\left.30^{\circ} \mathrm{C}\right),-1.4$; filled circles $\left(0.25 \mathrm{M}, 35^{\circ} \mathrm{C}\right),-1.57$; squares $\left(0.1 \mathrm{M}, 30^{\circ} \mathrm{C}\right),-2$; open triangles $\left(0.05 \mathrm{M}, 30^{\circ} \mathrm{C}\right),-4.4$; filled triangles $\left(0.05 \mathrm{M}, 35^{\circ} \mathrm{C}\right),-4.6$. After ref. 23 .

In order to confirm this explanation, we have studied reverse polymer-like micelles of soy-bean lecithin in isooctane, where ionic effects are absent (15). As expected, the observed behavior in similar to that of CTAB micelles at high salt. The measured exponent $\alpha\left(D_{s} \sim c^{-\alpha}\right)$ is $(1.35 \pm 0.05)$ in the reverse systems with molar water to surfactant ratio $w_{0}$ equal to 0,1 and 2. The $\alpha$ value is close to the value measured for CTAB at $\mathrm{c}_{\mathrm{s}}=0.25 \mathrm{M}$ and to the Cates value. 
For higher values of $\mathrm{w}_{0}(2.5)$, as well as for CTAB micelles with larger amounts of $\mathrm{KBr}(0.5 \mathrm{M}$ to $2 \mathrm{M})$ an unusual accelerated diffusion is observed (fig. 4). The recovery time is no longer proportional to $i^{2}$ but to $i^{\mu}$ with $\mu<2$. The recovery curve is however exponential. These features have been explained in terms of a Levy walk (26). The key point is that in some cases, a typical fluorescent probe has no time to explore the whole distribution of micellar size : at $t=0$, most of the probes are statistically in the longer micelles. As time goes on, due to micelle breakage and recombination, the probe explores shorter micelles and (provided that the diffusion coefficient is a sufficiently fast function of $L$ ) the diffusion is progressively accelerated.

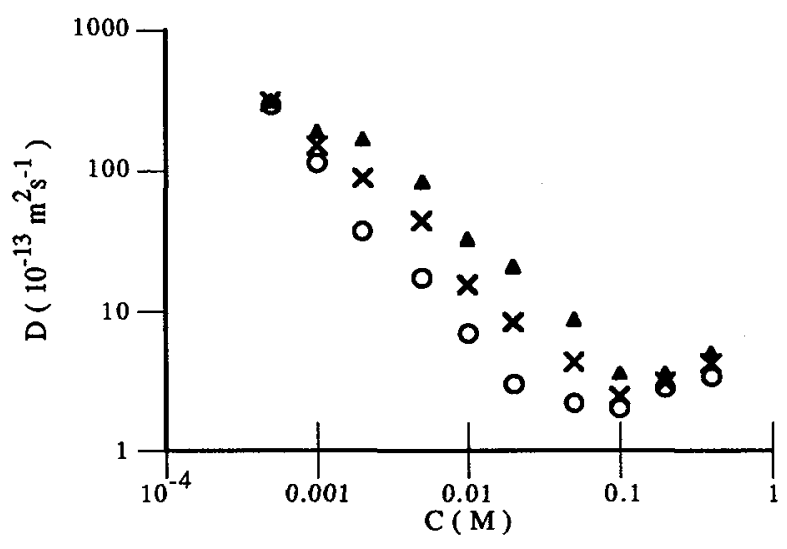

Figure 4. Effective diffusion coefficient determined at different length scales versus CTAB concentration in $2 \mathrm{M} \mathrm{KBr}$ brine. Triangles $: \mathrm{i}=100 \mu \mathrm{m}$, crosses $: \mathrm{i}=20$ $\mu \mathrm{m}$, circles : $\mathrm{i}=3 \mu \mathrm{m}$. Temperature is $40^{\circ} \mathrm{C}$. Part of data from ref. 26

Let us assume that the equilibrium size distribution is given by eq. 2 . The probability for a probe on a micelle of length $L$ to travel a length 1 is such that $: P(l) d l=P(L) d L$, and the total mean square displacement is :

$$
R^{2}=\int_{0}^{\infty} P(1) l^{2} d l
$$


Since $1^{2}=D(L) \tau_{b}(L) \sim L^{-3}$, the integral diverges. In fact, this integral is dominated by its lower bound $\mathrm{L}_{\min }$, determined by $\mathrm{N} \int_{\mathrm{min}}^{\infty} \mathrm{dL} P(\mathrm{~L})=1, \mathrm{~N}$ being the total number of breakage-recombination events. $N$ is related to the diffusion time t by $: t=N \int_{0}^{\infty} d L P(L) \tau_{b}(L)$. One thus finds $: R^{2}(t) \sim t \log t$. In order to obtain a measurable acceleration of the diffusion, $R^{2} \gg t$, one can for instance slightly modify the exponent for the reptation process : $D(L) \sim L^{-}$ $2 \beta$ and (or) slightly change the equilibrium size distribution of the micelles:

$$
P(L) \sim L^{1-2 \sigma} L^{-1+2 \sigma} \exp (-L / L)
$$

this can arise for instance, if scission near micellar ends is easier; then defining :

$$
\mu=2(1-\sigma) / \beta
$$

one finds for $\mu<2: R^{2}(t) \sim t^{2 / \mu}$. The diffusion is accelerated. It can also be shown that the fluorescence recovery signal is purely exponential with a decay rate proportional to $\mathrm{q}^{\mu}$.

The result $\mu<2$ is found for $\sigma>0$ (larger number of small micelles than predicted by eq. 2) and $\beta=1: D(L) \sim L^{-2}$ assuming that each individual chain undergo a pure reptation motion. It applies of course in a limited interval of micellar lengths : for small $L, L<\xi$, the chain is not entangled and $D(L) \sim L^{-1 / 2}$, leading to $\mu>2$. If $L>L_{c}=L\left(\tau_{b} / \tau_{\text {rep }}\right)^{1 / 3}$, the chain recombines in a time shorter than its disentanglement time, and one enters the Cates regime which again corresponds to $\mu>2$. Let us note that this regime corresponds to chains that are all larger than $\mathrm{L}_{\mathrm{c}}$.

The anomalous diffusion also ceases when the probe has the time to visit all the micelles, i.e. when $\mathrm{L}_{\min }=\xi$. In this long time limit, the largest contribution to the average diffusion comes from the short chains, and is : $D_{\text {short chains }} \sim D_{\text {rep }}(L)\left(L / L_{b}\right)^{2 \sigma}$, where $L_{b}$ is the chain length in a blob, to be compared with: $\mathrm{D}_{\text {Cates }}=\mathrm{D}_{\text {rep }}(\mathbf{L})\left(\mathbf{L} / \mathrm{L}_{\mathrm{c}}\right)$

In a number of experimental measurements, $\mu=1.5^{(26)}$, which for $\beta=1$ gives $\sigma=1 / 4$; the exponent $\alpha$ of the power law dependence of $D_{s}$ versus $\phi$ (or $c$ ) is then:

$$
\alpha=[v(2-3 \sigma)+(1-\sigma)] /(3 v-1)
$$

$\alpha$ is respectively $1.0,1.87$, or 2.75 for $v=1$ (stretched blobs, $\xi \sim l_{p}$ ), $v=3 / 5$ (swollen blobs), or $v=1 / 2$ (ideal blobs). The corresponding values for $\mathrm{D}_{\text {Cates }}$ are close, $0.83,1.58$, and 2.33 
respectively. One also notes that $\alpha$ is very sensitive to the chain conformation inside one blob. Since $\xi$ is never much larger than $1_{p}$ in the studied systems, the small differences between the measured and calculated exponents (for $v=3 / 5$ ) may arise from $v$ variations when $\phi$ increases and $\xi$ decreases. Let us note however, that in all the systems where $\xi$ has been measured, $v$ was found close to $3 / 5$ even at large surfactant concentration(21)(27)(28)(29).

In other systems, the micelles grow faster and entangle at smaller concentrations. This is the case for cetyltrimethylammonium salicylate (CTA Sal) when sodium salicylate is added (27)(30), and cetylpyridinium chlorate $\left(\mathrm{CP} \mathrm{ClO}_{3}\right)$ when sodium chlorate is added (29). In the first system the measured recovery curves are not exponential, probably meaning that the breaking time is longer than in CTAB micelles (31). In the $\mathrm{CPClO}_{3}$ system, the recovery curves are exponential, and the small concentration $\mathrm{D}_{\mathrm{S}}$ values are close to those in the CTAB system; at larger surfactant concentrations, there is a transition from the power law regime to a relatively flat plateau (fig. 5). This is in fact observed in most cases but the plateau level varies $D_{S} \sim 10^{-12}$ $\mathrm{m}^{2} / \mathrm{sec}$ for $\mathrm{CPClO}_{3}+1 \mathrm{M} \mathrm{NaClO}_{3}, \mathrm{D}_{\mathrm{s}} \sim 10^{-13} \mathrm{~m}^{2} / \mathrm{sec}$ for CTAB at high $\mathrm{KBr}$ content, $\mathrm{D}_{\mathrm{s}} \sim$ $10^{-14} \mathrm{~m}^{2} / \mathrm{sec}$ for lecithin micelles $\left(\mathrm{w}_{0}=2.5\right)$.

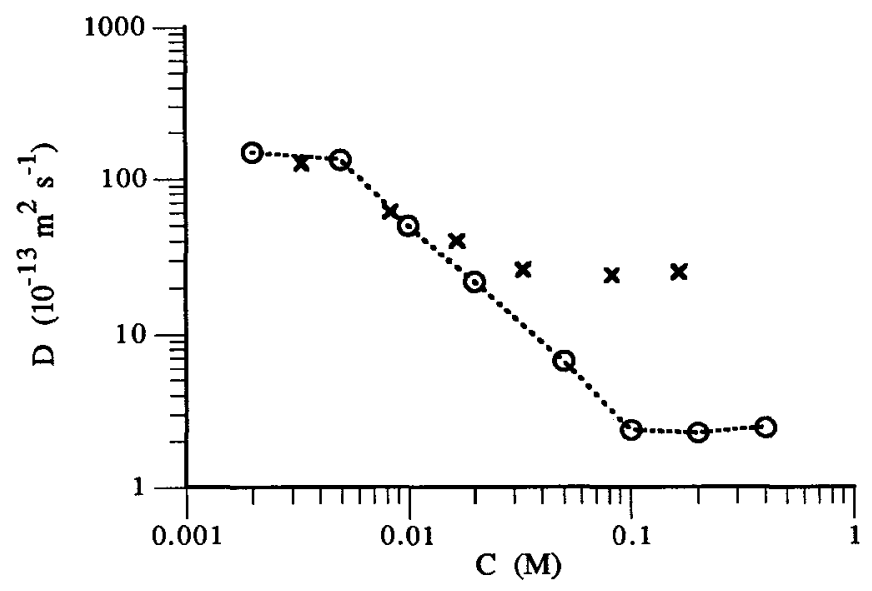

Figure 5. $\mathrm{Log}-\log$ plot of $\mathrm{D}_{\mathrm{S}}$ versus $\mathrm{C}$; circles: $\mathrm{CTAB}+0.5 \mathrm{M} \mathrm{KBr}$, crosses : $\mathrm{CPClO}_{3}+$ $1 \mathrm{M} \mathrm{NaClO}_{3}$.

In these concentration regions, $\xi$ is of order $l_{p}$, but as discussed before, the diffusion coefficient is expected to decrease further : $\alpha>0.83$. It is also conceivable that $\sigma$ increases, because in the concentrated regime, the probability for a chain to recombine shortly after a break 
is greater for a large chain because short chains diffuse away from each other faster. Using eq. 10 , one sees that $\alpha$ vanishes for $v=1$ and $\sigma=3 / 4$.

Another possibility is progressive branching. In the case of $\mathrm{CPClO}_{3}$ micelles, this is strongly suggested by the behaviour of the elastic modulus which does not follow the predictions for polymer-like systems (32). Let us note that in this case, the behaviour is independent of the dynamic processes (the value of $\tau_{b}$ for instance) because the modulus is a static property.

Finally when the micelles becomes long enough, the diffusion motion on the micelle itself can be faster than the reptation. When this occurs, the distance travelled by the micelle before a breakage-recombination event occurs is such as $L^{2}=D_{t} \tau$, where $D_{t}$ is the selfdiffusion coefficient along the micelle and $\tau=1 /(\mathrm{CL})$. Assuming that the motion of the probe at long times consists of random steps of mean spatial extent $\sqrt{\mathrm{LI}_{\mathrm{p}}}$ on the time scale $\tau$, one then finds

$$
D_{s}=D_{t}^{2 / 3} \tau_{b}^{-1 / 3} L^{-1 / 3} l_{p}
$$

For CTAB micelles, $D_{s}$ is aproximately constant above $c=100 \mathrm{mM}$; taking $D_{t}=10^{-11} \mathrm{~m}^{2} / \mathrm{s}, \tau_{b}$ $=0.1 \mathrm{sec}(24), l_{p} \sim 100 \AA$, and $L \sim 1 \mu \mathrm{m}$, a reasonable value at these concentrations, one finds $D_{s}$ $\sim 10^{-13} \mathrm{~m}^{2} / \mathrm{s}$, in good agreement with the measured value.

\section{Conclusion.}

The self-diffusion processes in systems of polymer-like micelles are extremely varied, especially in the semi-dilute region where these systems also exhibit viscoelastic behavior. One can observe reptation processes interrupted by breakage and recombination. In some cases this can lead to an accelerated diffusion, an experimental materialization of the Levy walk process. Although the experimental evidence is less conclusive, possible branching of the micelles and tracer diffusion along the micelles also seems to affect appreciably the diffusion behavior. A good understanding of the diffusion behavior is important to clarify the properties of these systems which are very promising for future practical applications in domains where a control of the rheological behaviour is required.

\section{References}

(1) A. Coniglio, E. Stanley, W. Klein, Phys. Rev. Lett. 42, 518 (1979).

(2) P.G. de Gennes, J. Phys. Lett., 40 , L-197 (1979).

(3) P.G. de Gennes "Scaling Concepts in Polymer Physics" Cornell Univ. Press 1979. 
(4) P.N. Pusey, W. van Megen, J. Phys. 44285 (1983).

(5) A.M. Cazabat, D. Chatenay, D. Langevin, J. Meunier, L. Léger, in "Surfactants in solution" Ed. K.L. Mittal, B. Lindman, Vol. 3, p.1729, Plenum Press, 1982.

(6) J. Davoust, P. Devaux, L. Léger, EMBO J. 101233 (1982).

(7) S. Cribier, L. Bourdieu, R. Vargas, A. Gulik, V. Luzzati, J. Phys. 51105 (1990).

(8) D. Chatenay, P. Guering, W. Urbach, A.M. Cazabat, D. Langevin, J. Meunier, L. Léger, B. Lindman, in "Surfactants in solution" Ed. K.L. Mittal, P. Bothorel, Vol. 6, p.1373, Plenum Press, 1984.

(9) E. Sackmann, J. Engelhardt, K. Fricke, H. Gaub, Colloids and Surfaces 10321 (1984)

(10) E.A.G. Aniansson, S.N. Wall, M. Almgren, H. Hoffmann, J. Kielman, W. Ulbricht, R. Zana, J. Lang, C. Tondre, J. Phys. Chem. 80, 905 (1976).

(11) A.Ott, W.Urbach, D.Langevin, H.Hoffmann, Langmuir, $\underline{8}, 345$ (1992)

(12) C. Tanford, "The hydrophobic effect", John Wiley, 1973.

(13) J. Israelachvili, D.J. Mitchell, B.W. Ninham, J. Chem. Soc. Faraday Transactions II, 72. 1525 (1976).

(14) M.E. Cates, S.J. Candau, J. Phys. Condens. Matter 2 ,6869 (1990).

(15) A. Ott, W. Urbach, D. Langevin, P. Schurtenberger, R. Scartazzini, P.L. Luisi, J. Phys. Condens. Matter 2. 5907 (1990).

(16) P. Vinson, Y. Talmon, J. Colloid Interface Sci. 133. 288 (1989).

(17) S.A.Safran, L.A.Turkevich, P.Pincus, J.Phys. Lett. 45, L-69 (1984)

(18) J.Appell, P.Basserau, J.Marignan, G.Porte, Prog. Colloid Polym. Sci., 81, 4173 (1990)

(19) P.Schurtenberger, L.J.Magid, S.M.King, P.Lindner, J.Phys.Chem. 25,4173 (1991)

(20) M.E. Cates, Macromolecules 202289 (1987) ; J. Phys. 49 , 1593 (1988).

(21) S.J. Candau, E. Hirsch, R. Zana, J. Colloid Interface Sci. 105 ,521 (1985).

(22) S.J. Candau, E. Hirch, R. Zana, M. Adam, J. Colloid Interface Sci. 122 ,430 (1988).

(23) R. Messager, A. Ott, D. Chatenay, W. Urbach, D. Langevin, Phys. Rev. Lett. 60, 1410 (1988).

(24) S.J. Candau, F. Merikhi, G. Waton, P. Lemaréchal, J. Phys. France 51977 (1990).

(25) S.A. Safran, P.A. Pincus, M.E. Cates, F.C. Mackintosh, J. Phys. France 51 503 (1990).

(26) A. Ott, J.P. Bouchaud, D. Langevin, W. Urbach, Phys. Rev. Lett. 65 ,2201 (1990); J. Phys. II France 1 , 1465 (1991).

(27) R.Makloufi, E.Hirsch, S.Candau, W.Banana-Limbélé, R.Zana, J.Phys.Chem., 93, 8095 (1989)

(28) P.Schurtenberger, R.Scartazzini, L.J.Magid, M.E.Leser, P.L.Luisi, J.Phys.Chem. 94, 3695 (1990)

(29) J.Appell, G.Porte, Europhys. Lett. 12185 (1990); Prog. Colloid Polym. Sci. $\underline{84}, 41$ (1991)

(30) H. Hoffmann, M. Löbe, H. Rehage in "Physics in amphiphiles : micelles, vesicles and microemulsions" Ed. V. Degiorgio, M. Corti, North Holland (1985).

(31) R. Messager, Thèse, Université de Paris 6, France, 1989.

(32) J. Appell, G. Porte, A. Khatory, F. Kern, S.J. Candau, J. Phys. II France 21045 (1992).S.J. Candau, these proceedings. 